\title{
Preparation and Characterization of Kynurenic Acid Occluded in Sol-Gel Silica and SBA-15 Silica as Release Reservoirs
}

\author{
Tessy López, ${ }^{1,2,3,4}$ Emma Ortiz, ${ }^{1}$ Esteban Gómez, ${ }^{1}$ \\ Verónica Pérez-de la Cruz, ${ }^{5}$ Paul Carrillo-Mora, ${ }^{6}$ and Octavio Novaro ${ }^{4,7}$ \\ ${ }^{1}$ Nanotechnology Laboratory, National Institute of Neurology and Neurosurgery, 14269 Mexico City, Mexico \\ ${ }^{2}$ Department of Chemical and Molecular Engineering, Tulane University, New Orleans, LA 70118, USA \\ ${ }^{3}$ Nanotechnology and Nanomedicine Laboratory, Autonomous Metropolitan University-Xochimilco, 04960 Mexico City, Mexico \\ ${ }^{4}$ Institute of Physics, National Autonomous University of Mexico, 01000 Mexico City, Mexico \\ ${ }^{5}$ Neurochemistry Department, National Institute of Neurology and Neurosurgery, 14269 Mexico City, Mexico \\ ${ }^{6}$ Neuroscience Department, Subdivision of Neurobiology, National Institute of Rehabilitation, 14389 Mexico City, Mexico \\ ${ }^{7}$ El Colegio Nacional, 06020 Mexico City, Mexico
}

Correspondence should be addressed to Emma Ortiz; emmal70@hotmail.com

Received 4 March 2014; Revised 9 May 2014; Accepted 9 May 2014; Published 29 May 2014

Academic Editor: Abdelwahab Omri

Copyright (C) 2014 Tessy López et al. This is an open access article distributed under the Creative Commons Attribution License, which permits unrestricted use, distribution, and reproduction in any medium, provided the original work is properly cited.

Kynurenic acid (KYNA) may have important therapeutic effects in neurological disorders; however, its use as a neuroprotective agent is restricted due to its very limited ability to cross the blood brain barrier (BBB). For this reason, we are looking for new alternatives for KYNA to reach the brain; one of them is using drug delivery systems. To obtain KYNA release reservoirs, KYNA molecules were hosted in two different silica materials. The different KYNA-silica materials were characterized by means of several physical techniques. The spectroscopic studies showed that KYNA molecules remained unchanged once hosted in silica materials. The surface area values of KYNA-silica samples were substantially lower than those for pure silica materials due to the addition of the drug. The electronic micrographs showed that the sol-gel KYNA-silica material consisted of aggregates of nanoparticles around $50 \mathrm{~nm}$ in size. On the other hand, the typical SBA-15 hexagonal arrangement was observed, even when hosting KYNA molecules. KYNA release profiles, carried out during approximately 300 hours, showed a first stage of fast drug release followed by a slow release phase. The experimental values fitted to the Peppas equation indicate that the release mechanism was controlled by Fickian diffusion.

\section{Introduction}

The kynurenine pathway is the main route of tryptophan catabolism (Figure 1) and it has been associated with several neurodegenerative diseases due to the neuroactive and redox properties of its metabolites. Kynurenine pathway metabolites can modulate different processes such as neurotransmission, inflammation, and immunological responses, and they can also control the redox and chemical environment. One of the most studied metabolites of the kynurenine pathway is kynurenic acid (KYNA), which is so far the only known endogenous NMDA receptor antagonist. L-kynurenine, a precursor of KYNA, has shown to be neuroprotective in models of neurotoxicity. However, KYNA specific effects are difficult to follow since it crosses the blood brain barrier with great difficulty. Therefore, only a small percentage of the drug reaches its target site and the rest can be responsible for some side effects. In addition, the treatment of several brain diseases becomes difficult due to the fact that many neurotherapeutic and neuroimaging contrast agents are unable to reach therapeutic levels in the brain [1]. Different methods have been developed to deliver drugs across the BBB (blood brain barrier) to treat brain diseases [2].

To address the problem of BBB penetration, the use of nanocarriers for drug encapsulation and delivery represents an alternative. Nanomaterials-based drug delivery systems 


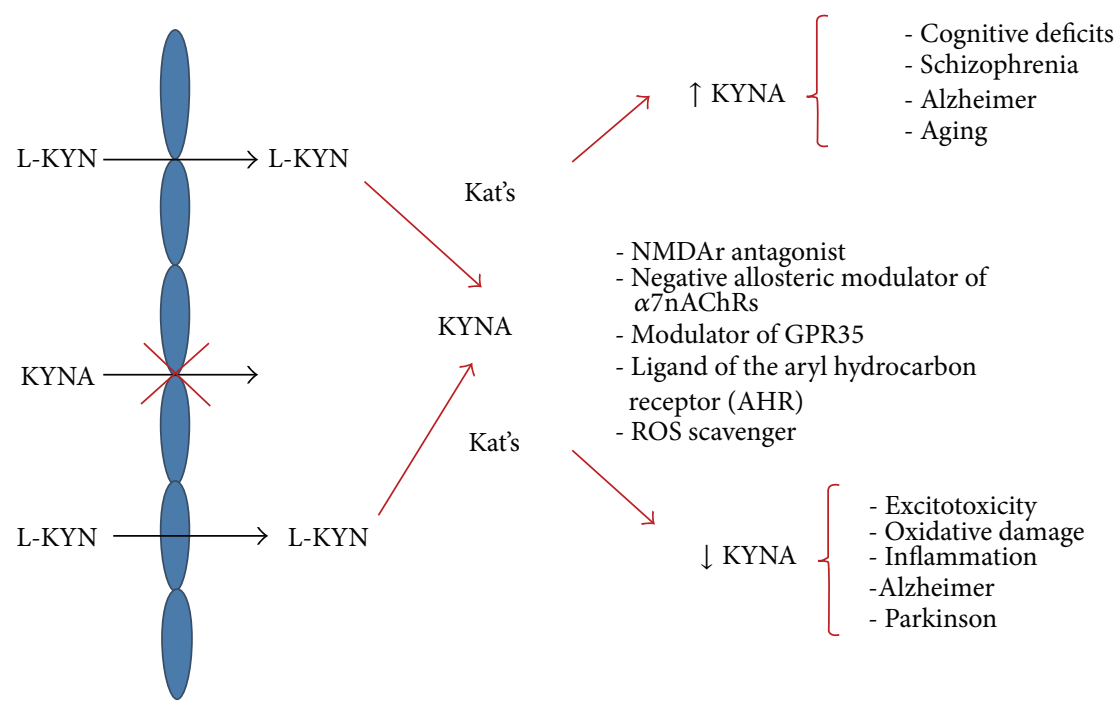

FIGURE 1: Mechanisms and neurodegenerative diseases related to KYNA.

offer many advantages such as allowing the drugs to be delivered in a site-specific way $[3,4]$, reducing the amount of drug and the number of administrations, protecting drugs against chemical and enzymatic degradation during their passage through the digestive tract, controlling drug release by modifying drug pharmacokinetics through physical or chemical interactions between drugs and carriers, and improving patients' compliance and comfort, $[5,6]$ as well as the possibility of using different routes of administration (implantation, inhalation, oral, and parenteral [7-10]).

Our research group has proposed using inorganic reservoirs composed of $\mathrm{TiO}_{2}$ and/or $\mathrm{SiO}_{2}$ as carriers for neurological drugs such as valproic acid, phenytoin, dopamine, Copaxone, and Interferone [11-14]. We have previously developed an implantable device, which releases the drug into a specific site in the brain (e.g., temporal lobe and striatum). We have already implanted that device in the temporal lobe of epileptic rats and in the striatum of parkinsonian rats. In both cases, we found not only a prolonged release of the neurological drug but also a high biocompatibility between the nanomaterials and rat brain tissue $[13,15]$. Silica, in its different shapes and sizes, is considered as a very promising material for hosting a variety of molecules including drugs, proteins, and other biomolecules, due to its physical properties such as large pores, high surface areas, bioresorbability, and biocompatibility [16-20].

Considering that KYNA has a wide range of potential applications in brain diseases [21-25] but it poorly penetrates the BBB [26], a mechanism to ensure an appropriate KYNA concentration within the brain in a controlled release manner is needed. In the present work, we are reporting the encapsulation of KYNA molecules in two different silica materials
(SBA-15 and silica prepared by the sol-gel route) with the purpose of releasing KYNA both under in vitro and in vivo conditions.

\section{Materials and Methods}

2.1. Silica-Based Materials Preparation. KYNA molecules were hosted in silica-based carriers using two different methods: (1) SBA-15 was impregnated with a KYNA solution and (2) KYNA molecules were added during the preparation of silica using the sol-gel method. Pure $\mathrm{SiO}_{2}$ prepared by the sol-gel method and SBA-15 without the drug were taken as references. 1:16 and 1:8 molar ratios of alkoxide: water and alkoxide : ethanol, respectively, were used.

Chemicals. Ethanol (ALPIMEX, 98 ${ }^{\circ}$ ), tetraethoxysilane [TEOS] (SIGMA-ALDRICH, 98\%), kynurenic acid (SIGMA, 98\%), and ammonium hydroxide (J. T. BAKER, 28-30\%).

Sol-Gel SiO ${ }_{2}\left(\right.$ Named $\left.\mathrm{SiO}_{2}\right) .70 \mathrm{~mL}$ of ethanol and $47 \mathrm{~mL}$ of deionized water were mixed and stirred for thirty minutes. Then, $37 \mathrm{~mL}$ of TEOS was added dropwise to the mixture over a period of four hours. Next, the final mixture was stirred at $30^{\circ} \mathrm{C}$ until the formation of the gel which was dried at $30^{\circ} \mathrm{C}$.

Sol-Gel $\mathrm{SiO}_{2}-\mathrm{KYNA}$ (Named $\mathrm{SiO}_{2}-\mathrm{KYNA}$ ). $400 \mathrm{mg}$ of KYNA was dissolved and stirred in $47 \mathrm{~mL}$ of deionized water. Next, five drops of $1 \mathrm{mM}$ ammonium hydroxide were added to complete the dissolution of KYNA. After, $70 \mathrm{~mL}$ of ethanol was added and $37 \mathrm{~mL}$ of TEOS was added dropwise during 


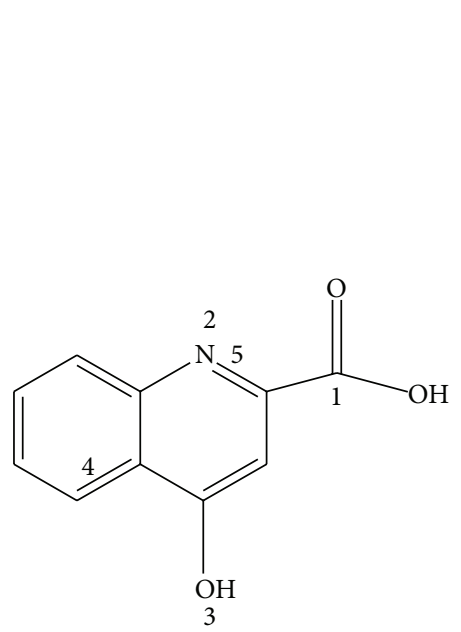

(a)

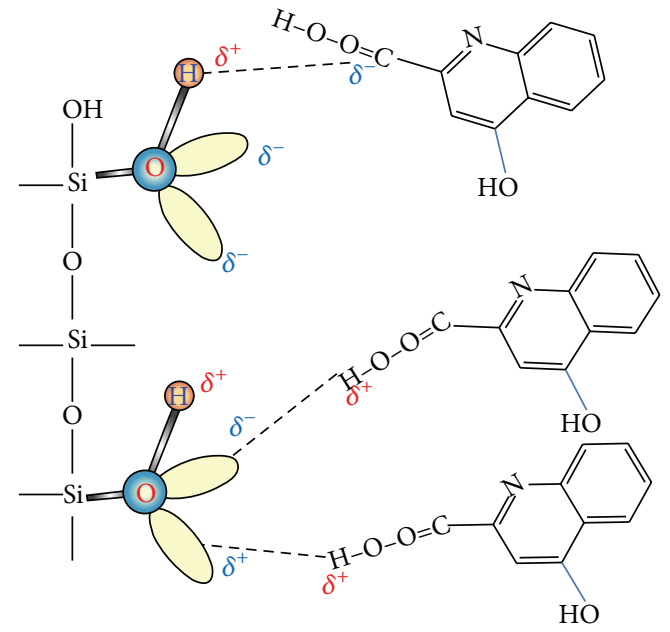

(b)

FIGURE 2: (a) Chemical structure of kynurenic acid and its functional groups: carboxyl (1), hydroxyl (3), amine (2), and C=C double bonds of aromatic rings (4). (b) Schematic of the interactions between silica surface and KYNA molecules. Dotted lines represent hydrogen bonds.

four hours. The final mixture was stirred at $30^{\circ} \mathrm{C}$ until the formation of the gel, which was dried at $30^{\circ} \mathrm{C}$.

SBA-15. We utilized the same previously reported method of preparation $[11,14]$. Our purpose was to compare the two methods used to host KYNA and their influence on the KYNA release profiles.

SBA-15-KYNA. $200 \mathrm{mg}$ of KYNA was dissolved in $25 \mathrm{~mL}$ of deionized water and basified by applying three drops of a $1 \mathrm{mM}$ ammonium hydroxide solution. Next, $5 \mathrm{~g}$ of SBA-15 was added to the KYNA solution. The SBA-15-KYNA mixture was stirred during 24 hours. Finally, the excess of water was evaporated.

\subsection{Characterization}

Fourier Transform Infrared Spectroscopy (FTIR). Each sample was mixed with potassium bromide to obtain a translucent wafer which was placed in an IRAffinity-1 Shimadzu spectrophotometer and measured in the medium infrared region $\left(4000-400 \mathrm{~cm}^{-1}\right)$.

Ultraviolet-Visible Spectroscopy Studies (UV-VIS). KYNA was dissolved in a $1 \mathrm{mM}$ sodium hydroxide solution and analyzed by UV-VIS spectroscopy in an S-3100 SCINCO spectrometer from 800 to $200 \mathrm{~nm}$. Similarly, the samples of KYNAcontaining silica were suspended in the alkaline solution before being analyzed.

$\mathrm{N}_{2}$ Adsorption-Desorption. The textural values from the $\mathrm{N}_{2}$ adsorption-desorption isotherm were obtained by using BelsorptII-BEL equipment. The surface area was calculated from the BET equation and the pore distribution and volumes were obtained from the BJH method. The samples were vacuumtreated at $30^{\circ} \mathrm{C}$ for $24 \mathrm{~h}$ prior to the $\mathrm{N}_{2}$ adsorption-desorption measurement.

Transmission Electronic Microscopy (TEM). The micrographs of the materials were obtained using a JEOL JEM-2100F electron microscope with a $200 \mathrm{kV}$ accelerated electron beam. The samples were placed over a cooper grid with holey carbon support film. Bright field technique was used.

2.3. "In Vitro" KYNA Release Test. $\mathrm{SiO}_{2}$-KYNA powder was compressed to form a tablet of approximately $3 \mathrm{mg}$, which was placed in a quartz cell containing $4 \mathrm{~mL}$ of deionized water. KYNA release was monitored by ultraviolet spectroscopy through the increment of absorbance intensity at $334 \mathrm{~nm}$. UV spectra were taken at predetermined times in an S-3100 SCINCO spectrometer. A standard curve of known KYNA concentrations versus absorbance was used to determine the amount of KYNA released.

\section{Results and Discussion}

3.1. FTIR Spectroscopy. $\mathrm{SiO}_{2}$ and $\mathrm{SiO}_{2}$-KYNA samples were characterized by infrared spectroscopy. KYNA structure (IUPAC name: 4-oxo- $1 \mathrm{H}$-quinoline-2-carboxylic acid) is shown in Figure 2(a); it contains the following functional groups: carboxyl, amine, hydroxyl, and a benzyl ring (numbers 1 to 4, resp., in Figure 2(a)), which are detectable by infrared spectroscopy. Figure 3 shows the FTIR spectra of $\mathrm{KYNA}, \mathrm{SiO}_{2}$, and $\mathrm{SiO}_{2}-\mathrm{KYNA}$ in the region of $4000-2500 \mathrm{~cm}^{-1}$. The first spectrum corresponds to KYNA. The signal at $3474 \mathrm{~cm}^{-1}$ was derived from the stretching vibrations of $-\mathrm{O}-\mathrm{H}$ bonds in the carboxyl group (number 1 in Figure 2(a)). This signal overlaps with the band of the $\mathrm{OH}$ bonded to the quinoline 


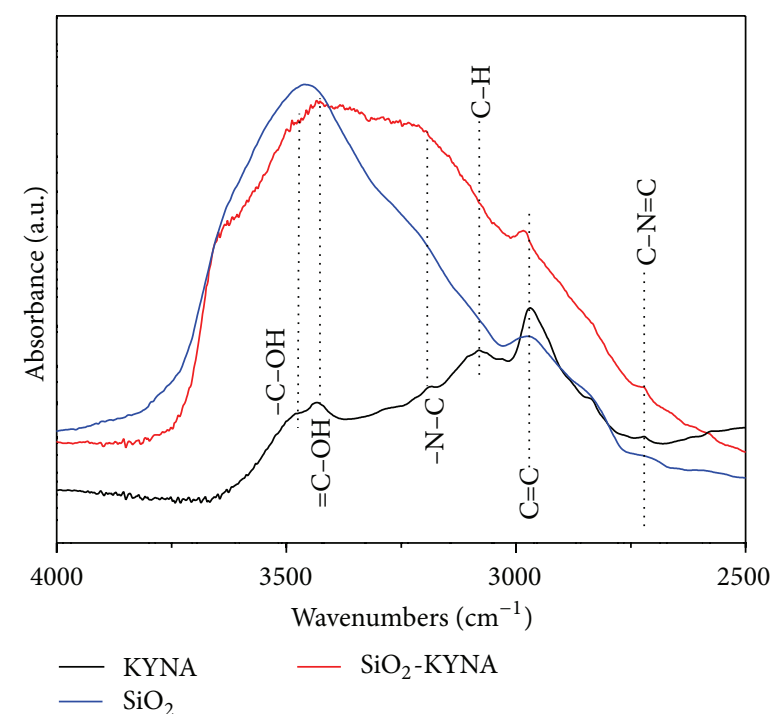

Figure 3: Infrared spectra of $\mathrm{KYNA}, \mathrm{SiO}_{2}$, and $\mathrm{SiO}_{2}-\mathrm{KYNA}$ samples in the low energy interval $\left(4000-2500 \mathrm{~cm}^{-1}\right)$.

ring (number 3 in Figure 2(a)), centered at $3432 \mathrm{~cm}^{-1}$. The small signal at $3193 \mathrm{~cm}^{-1}$ is associated with the $\mathrm{C}-\mathrm{H}$ bonds of the quinoline ring. The signal seen at $3076 \mathrm{~cm}^{-1}$ is attributed to the $\mathrm{C}-\mathrm{H}$ bonds of the quinoline ring, while the band at $2971 \mathrm{~cm}^{-1}$ corresponds to stretch vibrations of the $\mathrm{C}=\mathrm{H}$ bonds of benzyl and quinoline rings. Finally, the signal at $2718 \mathrm{~cm}^{-1}$ corresponds to the double bond $\mathrm{N}=\mathrm{C}$ in the quinoline ring (number 5 in Figure 2(a)).

The second spectrum corresponds to the $\mathrm{SiO}_{2}$ material, which has only two important signals: (a) a wide band centered at $3455 \mathrm{~cm}^{-1}$ that is commonly attributed to the $\mathrm{O}-\mathrm{H}$ bonds from the surface silanol groups $(\equiv \mathrm{Si}-\mathrm{OH})$ and $\mathrm{OHs}$ from water molecules adsorbed on the silica surface [27] and (b) a band located at $2968 \mathrm{~cm}^{-1}$, within the $\mathrm{C}-\mathrm{H}$ stretching vibrations region which suggests the presence of residual organic matter from the synthesis precursors.

Finally, the $\mathrm{SiO}_{2}$-KYNA spectrum consists of a wide band with small signals and others superimposed. The pattern of this spectrum is different from that of $\mathrm{SiO}_{2}$, indicating that the extra signals might be attributed to the hosted KYNA molecules because they occurred at the same positions as those of KYNA, as indicated by dotted lines (Figure 3). These results suggest that KYNA structure did not change during the process of silica synthesis; however, we noticed that the signal corresponding to the $\mathrm{O}-\mathrm{H}$ bond from the carboxyl group was shifted to a higher frequency $\left(3634 \mathrm{~cm}^{-1}\right)$. We attribute this displacement to the interactions between KYNA and silanol groups $(\equiv \mathrm{Si}-\mathrm{OH})$ on silica surface (Figure $2(\mathrm{~b})$ ). We have previously reported that drugs hosted in silica and titania materials interact with the surface of the oxides through their functional groups [11, 13]. Similarly, KYNA molecules and silica surface interact via hydrogen bonds and dipole-dipole forces, as shown in Figure 2(b). Hydrogen bonds can occur between hydrogen from the silanol group and oxygen from the KYNA carboxyl group or between

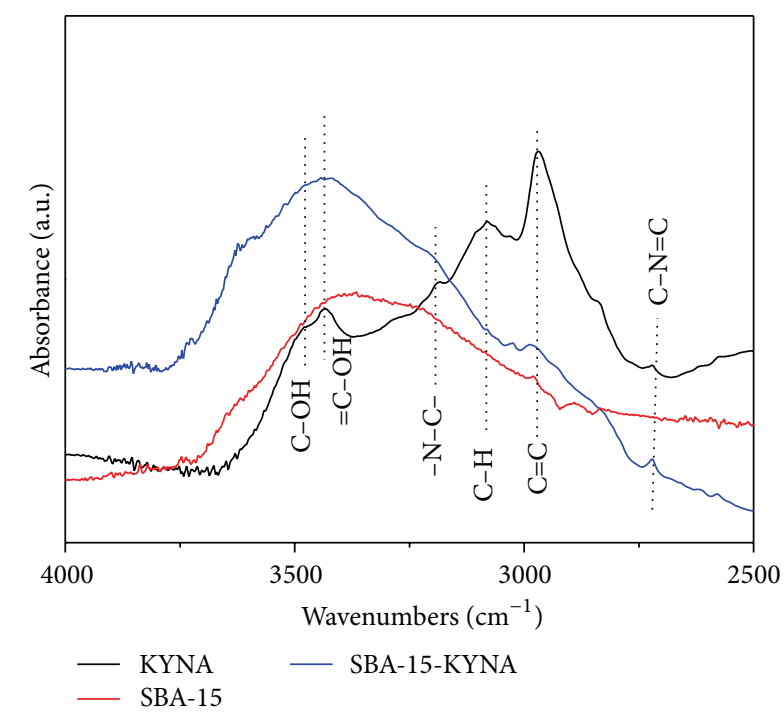

FIGURE 4: Infrared spectra of KYNA, SBA-15, and SBA-15-KYNA in the low energy interval $\left(4000-2500 \mathrm{~cm}^{-1}\right)$.

hydrogen from the KYNA carboxyl group and oxygen from the silanol group.

The spectra of SBA-15 and SBA-15-KYNA were also analyzed and reported in Figure 4. As expected, similar results were obtained for these samples. The dotted lines show that the signals corresponding to KYNA are also observed in the spectrum of SBA-15-KYNA. These results suggest that the structure of KYNA did not change during the impregnation procedure. Since SBA-15 is a silica-based material, having a different structural arrangement; it also has silanol groups that can interact with the guest drug molecules as we and others have previously reported $[28,29]$. Therefore, the interactions between KYNA and SBA-15 are similar to those showed in Figure 2(b).

The highest energy region of the spectrum is not displayed in the figures because KYNA signals were masked by the characteristic intense signal of silica.

3.2. Ultraviolet-Visible Spectroscopy. Ezaby et al. identified, by ultraviolet spectroscopy, three low-energy singlet-singlet absorption bands derived from KYNA due to $1 \mathrm{La}, 1 \mathrm{Lb}$, and $1 \mathrm{Bb}$ electronic transitions [30] from different sources: (a) electronic transitions from the $n p$ orbital of oxygen atoms from the $-\mathrm{OH}$ and $-\mathrm{COOH}$ groups, (b) electronic transitions from the $\mathrm{N}$ atom in the quinoline ring, and (c) $\pi-\pi^{*}$ electronic transitions from the double bonds in the benzyl ring. The intensity of the $1 \mathrm{La}$ and $1 \mathrm{Lb}$ bands can be affected by $\mathrm{pH}$ changes due to the ionizable groups bonded to the quinoline ring [31]. In order to identify KYNA, once released from the $\mathrm{SiO}_{2}, \mathrm{KYNA}$ and $\mathrm{SiO}_{2}-\mathrm{KYNA}$ were analyzed by UV-VIS spectroscopy in an alkaline solution. For this purpose, a $1 \mathrm{mM}$ sodium hydroxide solution was used to dissolve KYNA and to suspend $\mathrm{SiO}_{2}-\mathrm{KYNA}$ powder. The resulting UV-VIS spectra are displayed in Figure 5. KYNA spectrum shows a wide band between 280 and $360 \mathrm{~nm}$, centered at $\lambda=325 \mathrm{~nm}$. This band is consistent with the formation of the enolate anions from 


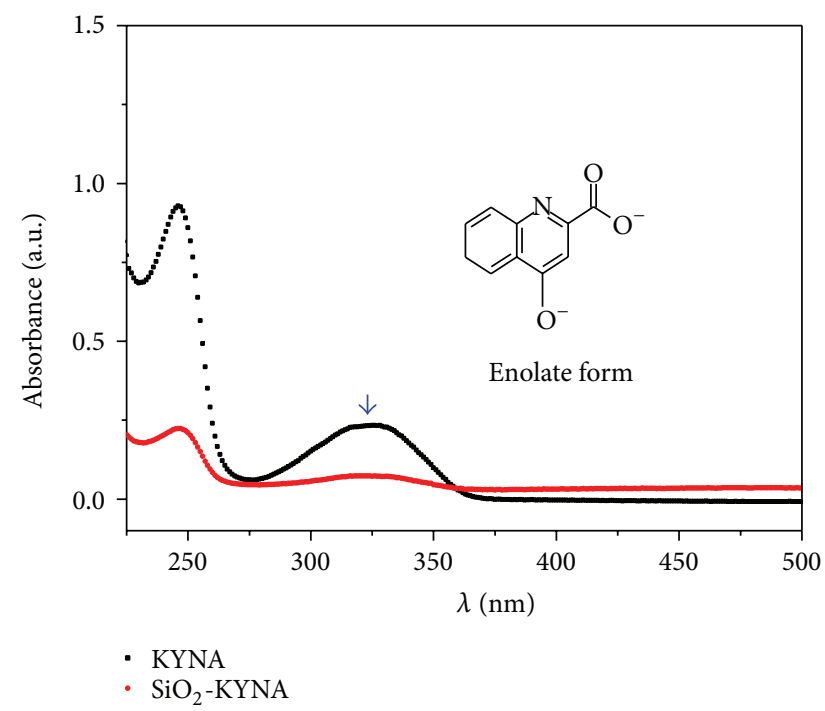

FIGURE 5: UV-VIS spectra of $\mathrm{KYNA}$ and $\mathrm{SiO}_{2}-\mathrm{KYNA}$ in alkaline solution. Under alkaline conditions KYNA assumes its enolate form due to the ionization of its hydroxyl groups.

the KYNA -OH groups as proposed by Pileni et al. (inset in Figure 5) [31]. The same band is observed on $\mathrm{SiO}_{2}-\mathrm{KYNA}$ spectrum suggesting that when KYNA is released from silica to the alkaline solution, it is immediately converted into its enolate form. The results obtained from the analysis of the UV spectra are consistent with those of the FTIR, meaning that KYNA molecules are not modified when added at the early stages of silica synthesis.

3.3. $\mathrm{N}_{2}$ Adsorption-Desorption. Unlike conventional methods for preparing silica, the sol-gel process allows the manipulation of the synthesis parameters $(\mathrm{pH}$, ethanol: TEOS ratio, water: TEOS ratio, and temperature, among others), thus facilitating the obtaining of porous silica materials with a high superficial area to host a variety of therapeutic compounds. Porosity and surface area of silica samples are commonly determined by nitrogen adsorption-desorption at $77 \mathrm{~K}$. The IUPAC classifies adsorption isotherms into six different categories [32], but only three of them are usually found in solid materials (type I, type II, and type IV).

Figure 6 shows the $\mathrm{N}_{2}$ adsorption-desorption isotherms of silica samples in the two different forms described above and their corresponding KYNA-containing forms. According to the IUPAC classification, the $\mathrm{SiO}_{2}$ isotherm can be classified as type IV, with a type-H4 hysteresis loop. The hysteresis loop was formed when relative pressure was between 0.4 and 0.9 , indicating a broad distribution of mesopores $(2 \mathrm{~nm}<$ pore size $<50 \mathrm{~nm}$ ) in the $\mathrm{SiO}_{2}$. The textural values obtained from the isotherms shown in Figure 6 are reported in Table 1. A high value of $492 \mathrm{~m}^{2} / \mathrm{g}$ was obtained for the surface area of the $\mathrm{SiO}_{2}$ sample, while its average pore size was $4.1 \mathrm{~nm}$. By contrast, the isotherm of $\mathrm{SiO}_{2}$-KYNA corresponds to a type I, with a narrow type-H3 hysteresis loop and large adsorption at high relative pressures, indicative of the presence of macropores (pore size $>50 \mathrm{~nm}$ ). The values of surface area

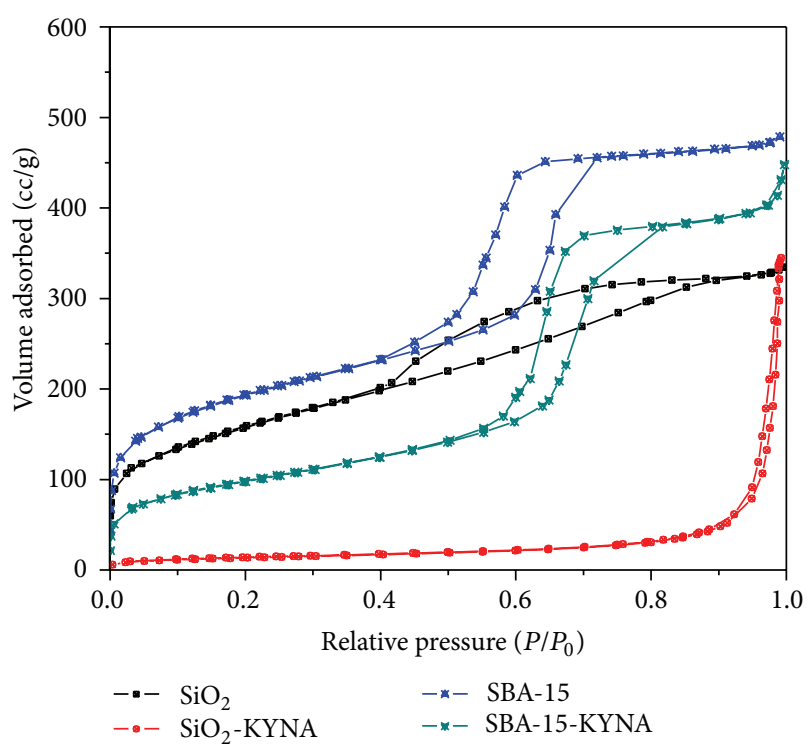

FIgURE 6: $\mathrm{N}_{2}$ adsorption-desorption isotherms of $\mathrm{SiO}_{2}, \mathrm{SiO}_{2}$ KYNA, SBA-15, and SBA-15-KYNA samples.

TABLE 1: Textural values of silica and KYNA-containing silica.

\begin{tabular}{lccc}
\hline Samples & $S_{\text {BET }}\left(\mathrm{m}^{2} / \mathrm{g}\right)$ & $D_{p}(\mathrm{~nm})$ & $V_{p}(\mathrm{cc} / \mathrm{g})$ \\
\hline $\mathrm{SiO}_{2}$ & 492 & 4.1 & 0.51 \\
$\mathrm{SiO}_{2}$-KYNA & 43 & 45.7 & 0.50 \\
$\mathrm{SBA}-15$ & 584 & 5.0 & 0.74 \\
SBA-15 KYNA & 314 & 8.3 & 0.65 \\
\hline
\end{tabular}

$S_{\mathrm{BET}}$ (surface area), $D_{p}$ (average pore diameter) and $V_{p}$ (pore volume).

and average pore diameter for this sample were $43 \mathrm{~m}^{2} / \mathrm{g}$ and $45.7 \mathrm{~nm}$, respectively, confirming the formation of macropores. It is noticeable that the presence of KYNA molecules in the silica network affected the textural values. The significant decrease in silica surface area is due to KYNA molecules occupying most of that space. We can suggest that large pores were formed when the nanoparticles were aggregated during the process of synthesis (Figure 7).

$\mathrm{N}_{2}$ adsorption-desorption isotherms of SBA-15 and SBA15 KYNA are showed in Figure 6 and the textural values are summarized in Table 1. Both samples resulted in type-IV isotherms, which are characteristic of mesoporous materials. The two samples exhibited the typical type-H1 hysteresis loops of this type of materials, due to a two-dimensional P6 mm structure formed by open cylindrical mesopores. Additionally, the isotherms displayed a sharp hysteresis loop in the $\mathrm{P} / \mathrm{P} 0$ range of $0.6-0.78$, revealing the uniformity of pore size. Both isotherms are similar in shape; however, the isotherms of SBA-15-KYNA show a smaller volume of adsorbed nitrogen, resulting in a decrease in the values of surface area and pore volume from $584 \mathrm{~m}^{2} / \mathrm{g}$ to $325 \mathrm{~m}^{2} / \mathrm{g}$ and from $0.74 \mathrm{cc} / \mathrm{g}$ to $0.65 \mathrm{cc} / \mathrm{g}$, respectively (Table 1 ). It is clearly deducible that KYNA caused this phenomenon due to (i) the space occupied by KYNA molecules in SBA-15 structure and (ii) KYNA molecules adsorbed in the channels without filling 


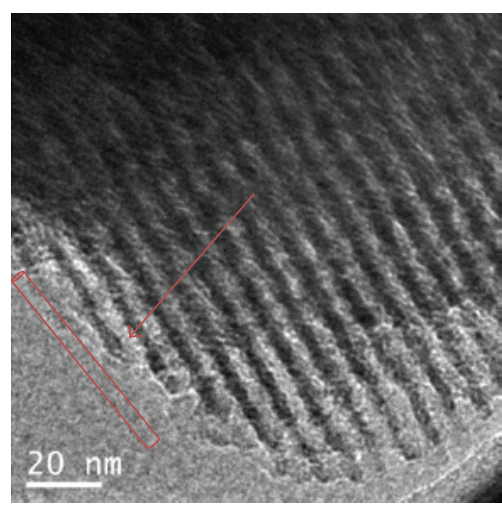

(a)

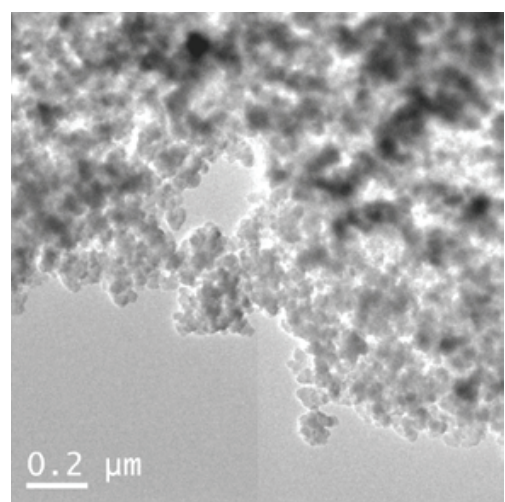

(d)

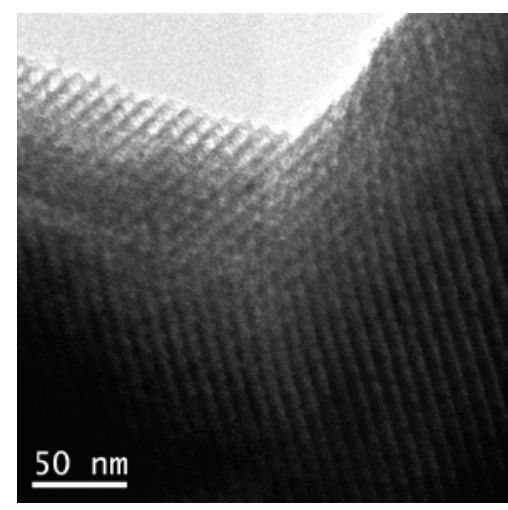

(b)

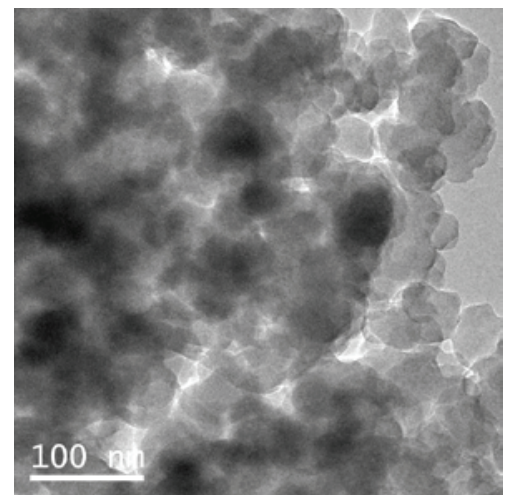

(e)

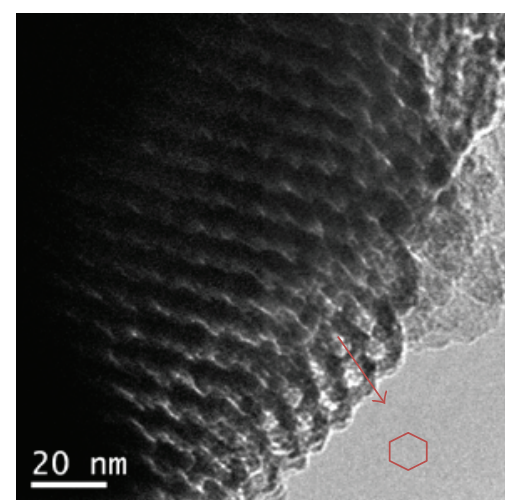

(c)

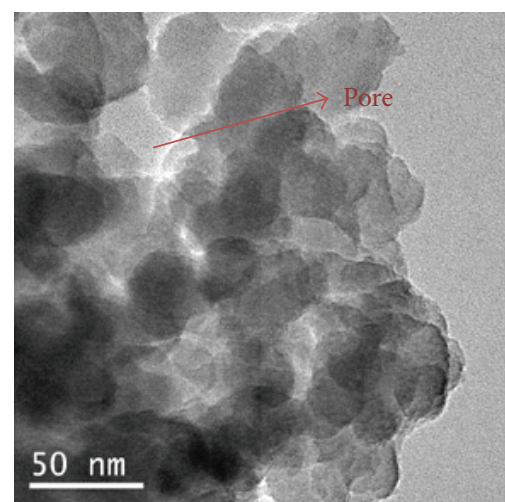

(f)

FIGURE 7: Micrographs from TEM studies of the silica-based materials. Cylindrical tubes with hexagonal mouths were observed in SBA-15KYNA samples $(\mathrm{a}-\mathrm{c})$, while aggregated nanoparticles and large spaces were present in $\mathrm{SiO}_{2}-\mathrm{KYNA}_{\text {samples. }}$

them totally. It is possible to state that KYNA did not affect the morphological structure of SBA-15 and its own structure was not altered once hosted in the SBA-15 material.

3.4. Transmission Electronic Micrographs (TEM) Analyses. The micrographs of $\mathrm{SiO}_{2}$-KYNA and SBA-15-KYNA are presented in Figure 7. As is shown in Figures 7(a)-7(c), SBA15-KYNA material has regular hexagonal arrays of cylindrical channels after KYNA was occluded. The channels have an average diameter of about $10 \mathrm{~nm}$ with a wall thickness of about $5 \mathrm{~nm}$. It is clearly seen that the mesoporous channels are maintained without any change after being loaded with the drug. These results agree with the nitrogen adsorptiondesorption measurements. The classical nanoparticles aggregates on sol-gel silica-KYNA were produced and are shown in Figures 7(d)-7(f). The nanoparticles show an amorphous formation, characteristic of silica nanoparticles, with an average particle size between 20 and $40 \mathrm{~nm}$. There can be spotted some pores through the sample with different pore sizes, mesopores with an average size similar to the particle size, $20-40 \mathrm{~nm}$, Figure $7(\mathrm{f})$, and macropores larger than $50 \mathrm{~nm}$ as can been seen in Figure 7(d). This corroborates the results obtained by $\mathrm{N} 2$ adsorption-desorption, which indicate the presence of mesopores and macropores.

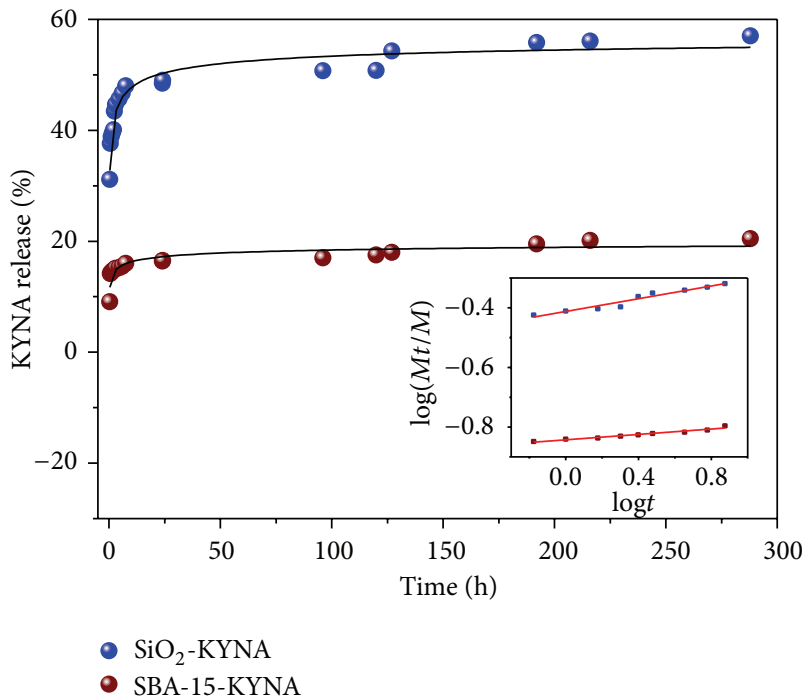

FIGURE 8: KYNA release profiles from sol-gel silica and SBA-15. The inset shows the experimental values fitted to the Peppas equation from which the $n$ values were determined.

3.5. "In Vitro" KYNA Release Tests. Figure 8 displays the KYNA release profiles from the two different silica materials. 


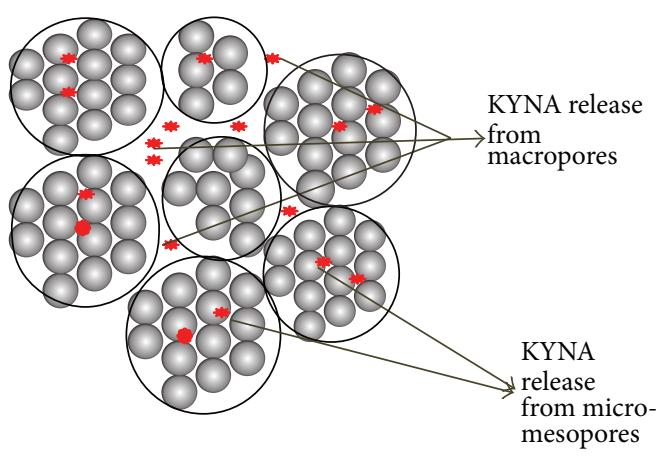

(a)

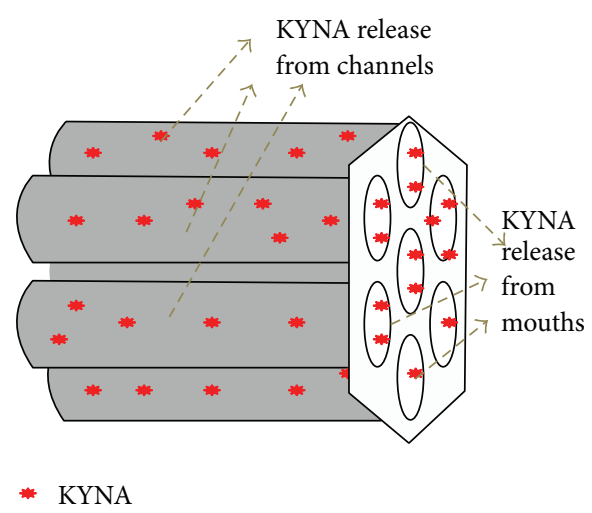

(b)

FIGURE 9: Proposed mechanism of KYNA release from macropores in sol-gel silica and from channels in SBA-15.

Both samples presented first-order release kinetics with an initial stage of fast release followed by a very slow release phase. A $50 \%$ of KYNA was released from $\mathrm{SiO}_{2}-\mathrm{KYNA}$ sample during the first $24 \mathrm{~h}$. This initial fast release can be associated with the pore size of $\mathrm{SiO}_{2}$-KYNA since it has the largest pore size $(45.7 \mathrm{~nm}$, Table 1$)$. It is possible that KYNA molecules could have easily left the silica because during the formation of the gel large pores were formed by the agglomeration of the particles, leaving most of KYNA molecules trapped in the macropores and then allowing them to be massively released, as shown in Figure 9(a). After that, in the slower diffusion stage, the drug was released from the smallest pores, mesopores and/or micropores, into the medium.

A $20 \%$ of KYNA was released from the SBA- 15 material within a period of $24 \mathrm{~h}$. This percentage is due to the fact that KYNA adsorbed in the hexagonal mounts of the SBA structure was released first as shown in Figure 9(b). After this time, KYNA release was slower because more time is needed for the molecules to diffuse through the channels composing SBA-15 structure.

In order to determine the diffusion mechanism of KYNA from the silica materials, the experimental data were fitted to Peppas' equation as we previously reported [14].

The logarithmic form of Peppas' equation is

$$
\log \left(\frac{M t}{M}\right)=\log k+n \log t
$$

where $M t / M$ is the fraction of drug released at time $t ; n$ is the diffusion exponent, which explains the transport mechanism of the drug through the oxide; and $k$ is the kinetic constant incorporating structural and geometric characteristics of the delivery system. Regarding $n$ values, if $n \leq 0.45$, the release mechanism follows Fickian diffusion; if $0.45<n<0.89$, the release occurs by non-Fickian diffusion; and if $n=1$, it is a zero order process.

The $n$ values were obtained from the slopes of the graphs inserted in Figure 8; $n$ was 0.4122 and 0.0452 for $\mathrm{SiO}_{2}$-KYNA and SBA-15-KYNA, respectively. As both values are less than 0.45 , then Fickian diffusion controls the release process.

\section{Conclusions}

(1) KYNA molecules were successfully occluded in solgel silica-based materials without undergoing any change in their structure.

(2) The morphology of SBA-15 was not affected by the incorporation of KYNA molecules.

(3) Textural properties were affected by the occlusion of KYNA molecules in both silica materials. In comparison with the corresponding empty silica, the surface area and the pore volume of $\mathrm{SiO}_{2}$-KYNA and SBA15-KYNA were reduced due to the space occupied by KYNA molecules.

(4) KYNA promoted the formation of macropores in solgel silica sample.

(5) KYNA release was faster from sol-gel silica than from SBA-15 due to the macropores in the former.

(6) The experimental values fitted to the big Peppas equation indicate that the release mechanism was controlled by Fickian diffusion.

\section{Conflict of Interests}

The authors declare that there is no conflict of interests regarding the publication of this paper.

\section{Acknowledgments}

This work was supported by ISN CAEN award and CONACYT Grant no. 183867.

\section{References}

[1] J. Kreuter, D. Shamenkov, V. Petrov et al., "Apolipoproteinmediated transport of nanoparticle-bound drugs across the blood-brain barrier," Journal of Drug Targeting, vol. 10, no. 4, pp. 317-325, 2002.

[2] G. Miller, "Drug targeting: breaking down barriers," Science, vol. 297, no. 5584, pp. 1116-1118, 2002. 
[3] G. Modi, V. Pillay, Y. E. Choonara, V. M. K. Ndesendo, L. C. du Toit, and D. Naidoo, "Nanotechnological applications for the treatment of neurodegenerative disorders," Progress in Neurobiology, vol. 88, no. 4, pp. 272-285, 2009.

[4] A. V. Kabanov and H. E. Gendelman, "Nanomedicine in the diagnosis and therapy of neurodegenerative disorders," Progress in Polymer Science, vol. 32, no. 8-9, pp. 1054-1082, 2007.

[5] T. M. Allen and P. R. Cullis, "Drug delivery systems: entering the mainstream,” Science, vol. 303, no. 5665, pp. 1818-1822, 2004.

[6] P. Kortesuo, M. Ahola, S. Karlsson, I. Kangasniemi, J. Kiesvaara, and A. Yli-Urpo, "Sol-gel processed sintered silica xerogel as a carrier in controlled drug delivery," Journal of Biomedical Material Research, vol. 44, pp. 162-167, 2000.

[7] M. Vallet-Regí, M. Colilla, and I. Izquierdo-Barba, "Bioactive mesoporous silicas as controlled delivery systems: application in bone tissue regeneration," Journal of Biomedical Nanotechnology, vol. 4, no. 1, pp. 1-15, 2008.

[8] S. Giri, B. G. Trewyn, M. P. Stellmaker, and V. S. Y. Lin, "Stimuliresponsive controlled-release delivery system based on mesoporous silica nanorods capped with magnetic nanoparticles," Angewandte Chemie, vol. 44, no. 32, pp. 5038-5044, 2005.

[9] M. Signoretto, E. Ghedini, V. Nichele, F. Pinna, V. Crocell, and G. Cerrato, "Effect of textural properties on the drug delivery behaviour of nanoporous $\mathrm{TiO}_{2}$ matrices," Microporous and Mesoporous Materials, vol. 139, no. 1-3, pp. 189-196, 2011.

[10] Y. Zhang, J. Zhang, T. Jiang, and S. Wang, "Inclusion of the poorly water-soluble drug simvastatin in mesocellular foam nanoparticles: drug loading and release properties," International Journal of Pharmaceutics, vol. 410, no. 1-2, pp. 118-124, 2011.

[11] T. López, E. Ortiz, D. Meza et al., "Controlled release of phenytoin for epilepsy treatment from titania and silica based materials," Materials Chemistry and Physics, vol. 126, no. 3, pp. 922929, 2011.

[12] T. Lopez, E. Ortiz, R. Alexander-Katz et al., "The effect of water on particle size, porosity and the rate of drug release from implanted titania reservoirs," Journal of Biomedical Materials Research B, Applied Biomaterials, vol. 93, no. 2, pp. 401-406, 2010.

[13] T. López, J. L. Bata-García, D. Esquivel et al., “Treatment of parkinson's disease: nanostructured sol-gel silica-dopamine reservoirs for controlled drug release in the central nervous system," International Journal of Nanomedicine, vol. 6, no. 1, pp. 19-31, 2011.

[14] T. López, E. Ortiz-Islas, M. López, J. Flores, and T. Corona, "Occlusion of INTERFERON and COPAXONE on SBA-15 silica reservoirs for their use in the treatment of demyelization diseases," Journal of Nanomaterials \& Molecular Nanotechnology, vol. 2, no. 4, pp. 1-6, 2013.

[15] T. López, E. Ortiz, P. Quintana, and R. D. González, "A nanostructured titania bioceramic implantable device capable of drug delivery to the temporal lobe of the brain," Colloids and Surfaces A: Physicochemical and Engineering Aspects, vol. 300, no. 1-2, pp. 3-10, 2007.

[16] E. Verraedt, M. Pendela, E. Adams, J. Hoogmartens, and J. A. Martens, "Controlled release of chlorhexidine from amorphous microporous silica," Journal of Controlled Release, vol. 142, no. 1, pp. 47-52, 2010.

[17] H. Guo, H. Qian, S. Sun et al., "Hollow mesoporous silica nanoparticles for intracellular delivery of fluorescent dye," Chemistry Central Journal, vol. 5, no. 1, article 1, 2011.
[18] Y. Zhu, W. Meng, H. Gao, and N. Hanagata, "Hollow mesoporous silica/poly(l-lysine) particles for codelivery of drug and gene with enzyme-triggered release property," Journal of Physical Chemistry C, vol. 115, no. 28, pp. 13630-13636, 2011.

[19] M. S. Bhattacharyya, P. Hiwale, M. Piras et al., "Lysozyme adsorption and release from ordered mesoporous materials," Journal of Physical Chemistry C, vol. 114, no. 47, pp. 19928-19934, 2010.

[20] J. Ho, Y. Huang, M. K. Danquah, H. Wang, and G. M. Forde, "Synthesis of biodegradable polymer-mesoporous silica composite microspheres for DNA prime-protein boost vaccination," European Journal of Pharmaceutical Sciences, vol. 39, no. 5, pp. 412-420, 2010.

[21] T. W. Stone, "Development and therapeutic potential of kynurenic acid and kynurenine derivatives for neuroprotection," Trends in Pharmacological Sciences, vol. 21, no. 4, pp. 149-154, 2000.

[22] T. W. Stone and L. G. Darlington, "Endogenous kynurenines as targets for drug discovery and development," Nature Reviews Drug Discovery, vol. 1, no. 8, pp. 609-620, 2002.

[23] J. Reyes-Ocampo, R. Lugo-Huitrón, D. González-Esquivel et al., "Kynurenines with neuroactive and redox properties: relevance to aging and brain diseases," Oxidative Medicine and Cellular Longevity, vol. 2014, Article ID 646909, 22 pages, 2014.

[24] S. Erhardt, L. Schwieler, C. Emanuelsson, and M. Geyer, "Endogenous kynurenic acid disrupts prepulse inhibition," Biological Psychiatry, vol. 56, no. 4, pp. 255-260, 2004.

[25] R. M. Kamiński, E. Zieliński, A. Dekundy, G. van Luijtelaar, and W. A. Turski, "Deficit of endogenous kynurenic acid in the frontal cortex of rats with a genetic form of absence epilepsy," Polish Journal of Pharmacology, vol. 55, no. 5, pp. 741-746, 2003.

[26] S. Fukui, R. Schwarcz, S. I. Rapoport, Y. Takada, and Q. R. Smith, "Blood-brain barrier transport of kynurenines: implications for brain synthesis and metabolism," Journal of Neurochemistry, vol. 56, no. 6, pp. 2007-2017, 1991.

[27] D. L. Wood and E. M. Rabinovich, "Study of alkoxide silica gels by infrared spectroscopy," Applied Spectroscopy, vol. 43, no. 2, pp. 263-267, 1989.

[28] T. Lopez, E. Ortiz, R. Alexander-Katz, E. Basaldella, and X. Bokhimi, "Cortisol controlled release by mesoporous silica," Nanomedicine: Nanotechnology, Biology, and Medicine, vol. 5, no. 2, pp. 170-177, 2009.

[29] T. López, E. Krötzsch, E. Ortiz-Islas et al., "Release properties and acute biosecurity determination of collagen-polyvinylpyrrolidone loaded in ordered mesoporous silica," Key Engineering Materials, vol. 391, pp. 169-184, 2009.

[30] E. Ezaby, M. S. T. M. Salem, M. Osman, and M. A. Makhyou, "Spectral studies on some quinoline derivatives of triptophane metabolities," Indian Journal of Chemistry, vol. 11, pp. 1142-1145, 1973.

[31] M. P. Pileni, M. Giraud, and R. Santus, "Kynurenic acid: I. Spectroscopic properties," Photochemistry and Photobiology, vol. 30, no. 2, pp. 251-256, 1979.

[32] K. S. W. Sing, D. H. Everett, R. A. Haul et al., "Reporting physisorption data for gas/solid systems with special reference to the determination of surface area and porosity," Pure and Applied Chemistry, vol. 57, no. 4, pp. 603-619, 1985. 

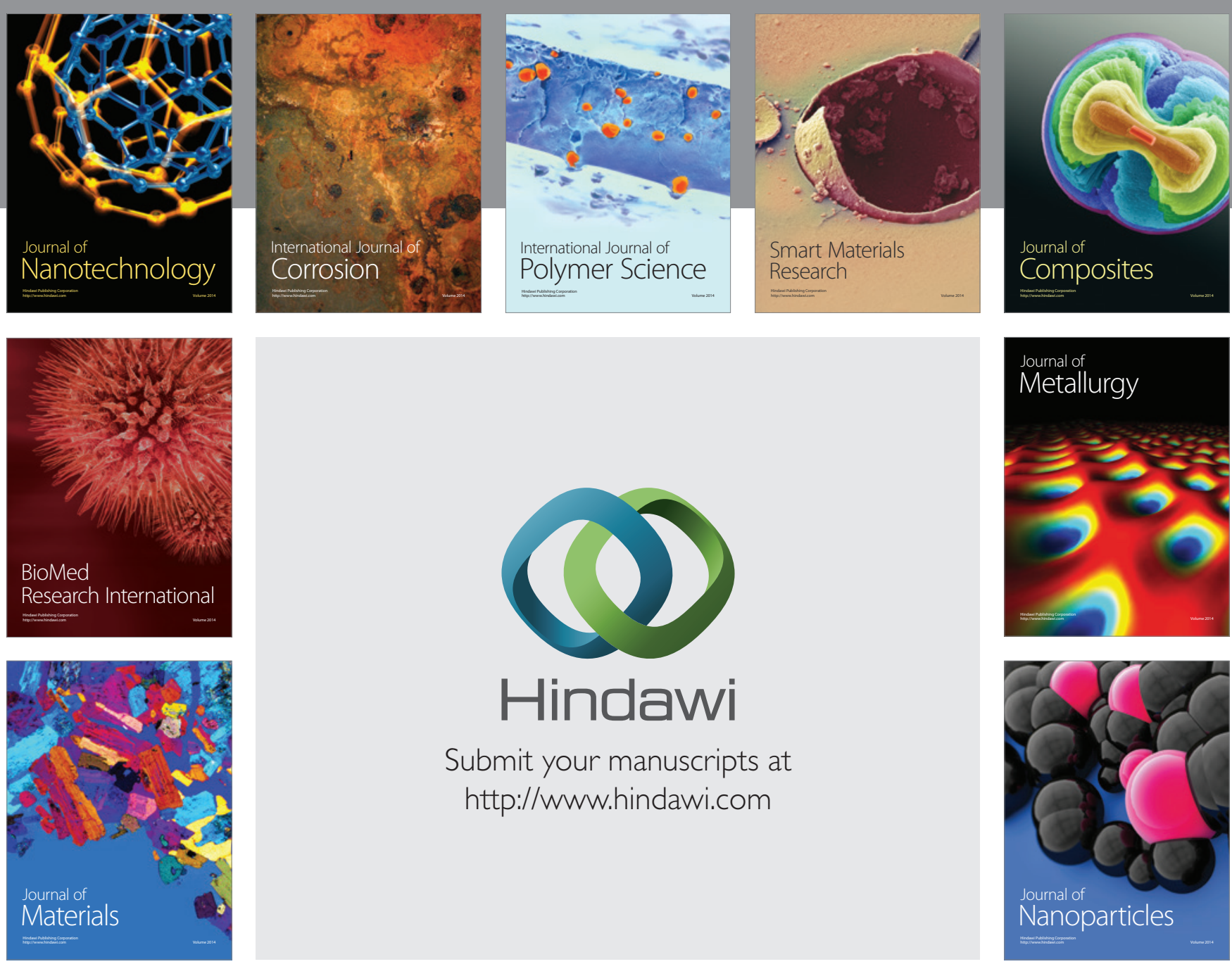

Submit your manuscripts at http://www.hindawi.com
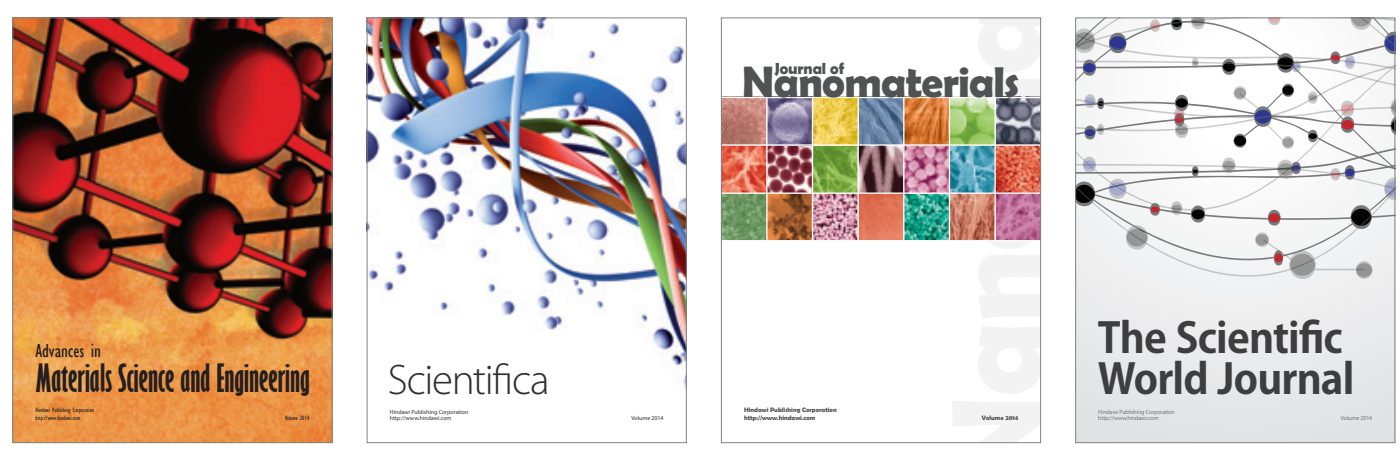

\section{The Scientific World Journal}
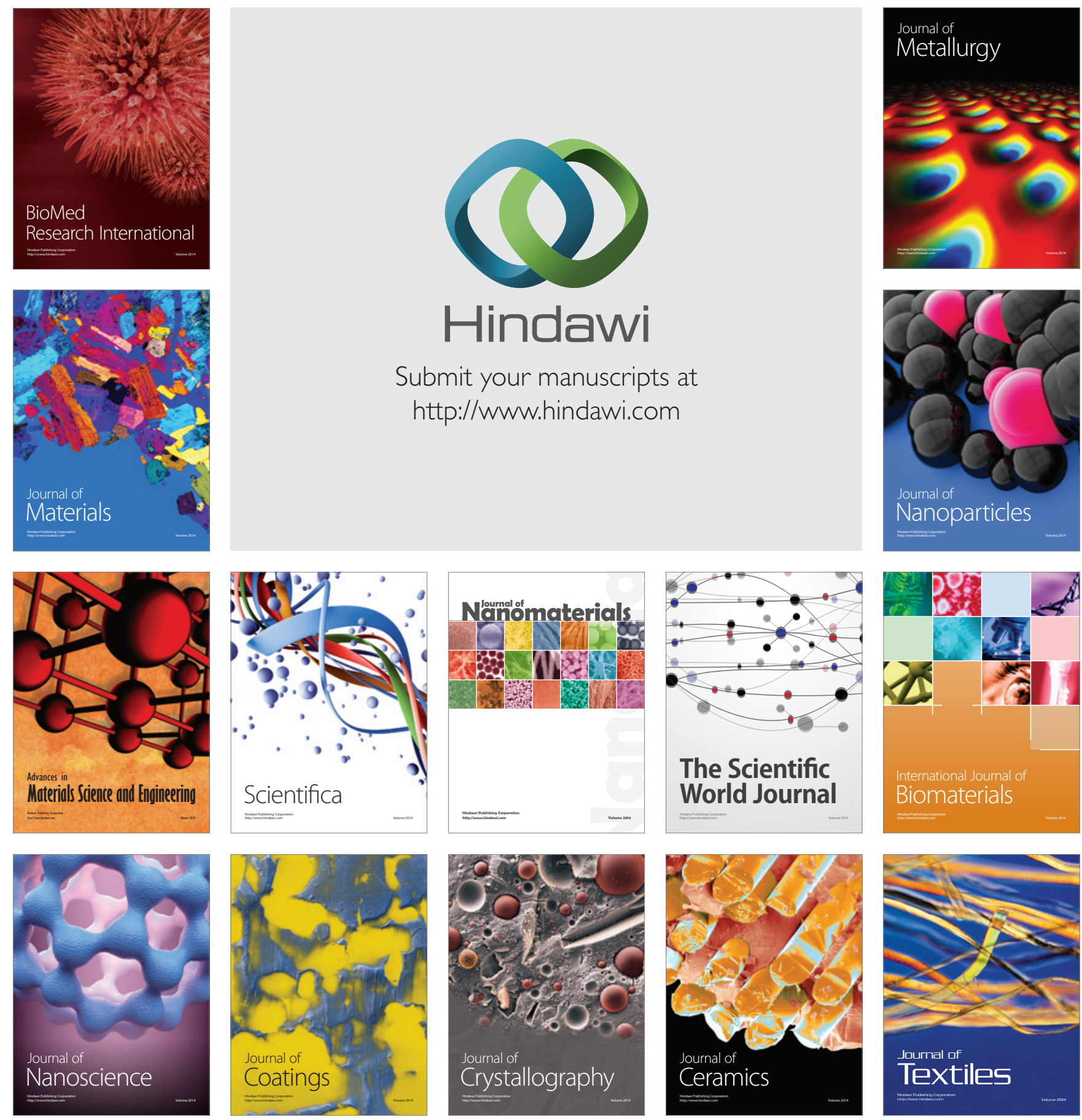